\title{
Zier-Spargel (Asparagus densiflorus)
}

\author{
Friedrich E. BeYHL
}

\begin{abstract}
The south African Asparagus densiflorus is a wide-spread ornamental plant for indoor cultivation. Its properties are described here.

\section{Zusammenfassung}

Asparagus densiflorus, eine südafrikansiche Spargel-Art, ist in Mitteleuropa eine weit verbreitete Zimmerpflanze. Sie wird hier genauer vorgestellt.
\end{abstract}

\section{Eine beliebte Zierpflanze}

Die Gattung Asparagus (Spargel) stammt aus tropischen und subtropischen Regionen der Alten Welt, allein in Madagaskar gibt es fünf Arten. Einige von ihnen werden als Zierpflanzen kultiviert.

Vielen Blumenfreunden ist allerdings nicht bewusst, dass es sich bei der beliebten und weit verbreiteten Zierpflanze, die gärtnerisch als 'Asparagus' oder 'Sprengeri' bezeichnet wird, um einen Verwandten unseres Gemüse-Spargels (Asparagus officinalis) handelt. Diese Spargel-Art hieß früher Asparagus sprengeri Regel, heute wird sie als Asparagus densiflorus (KUNTH) Jessop bezeichnet, was allerdings noch diskutiert wird (Jessor 1966, Green 1986, BatcheLOR \& SCOTT 2006).

Wie viele Vertreter der Gattung Aparagus ist Zier-Spargel eine Klimmpflanze, die sich an ihrer Stütze mit Hilfe stacheliger Organe festhält und in die Höhe wächst. In seiner Heimat Südafrika, aber auch als Kulturpflanze im Mittelmeergebiet, erreicht Zier-Spargel erstaunliche Dimensionen: In Athen traf der Autor ein Exemplar an, das im Freien an einer Hauswand ausgepflanzt war und an dieser bis über $15 \mathrm{~m}$ hoch wuchs. Auch im Frankfurter Palmengar-

Abb. 1 (oben): Blütenstand von Asparagus densiflorus. An den Blütenknospen bemerkt man deutlich die schuppenförmigen Tragblätter und die „gestielten Stiele“ sowie zwischen diesen an einigen Stellen Phyllokladien anstatt Blüten.

Abb. 2 (unten): Aufgeblühter Blütenstand von Asparagus densiflorus.
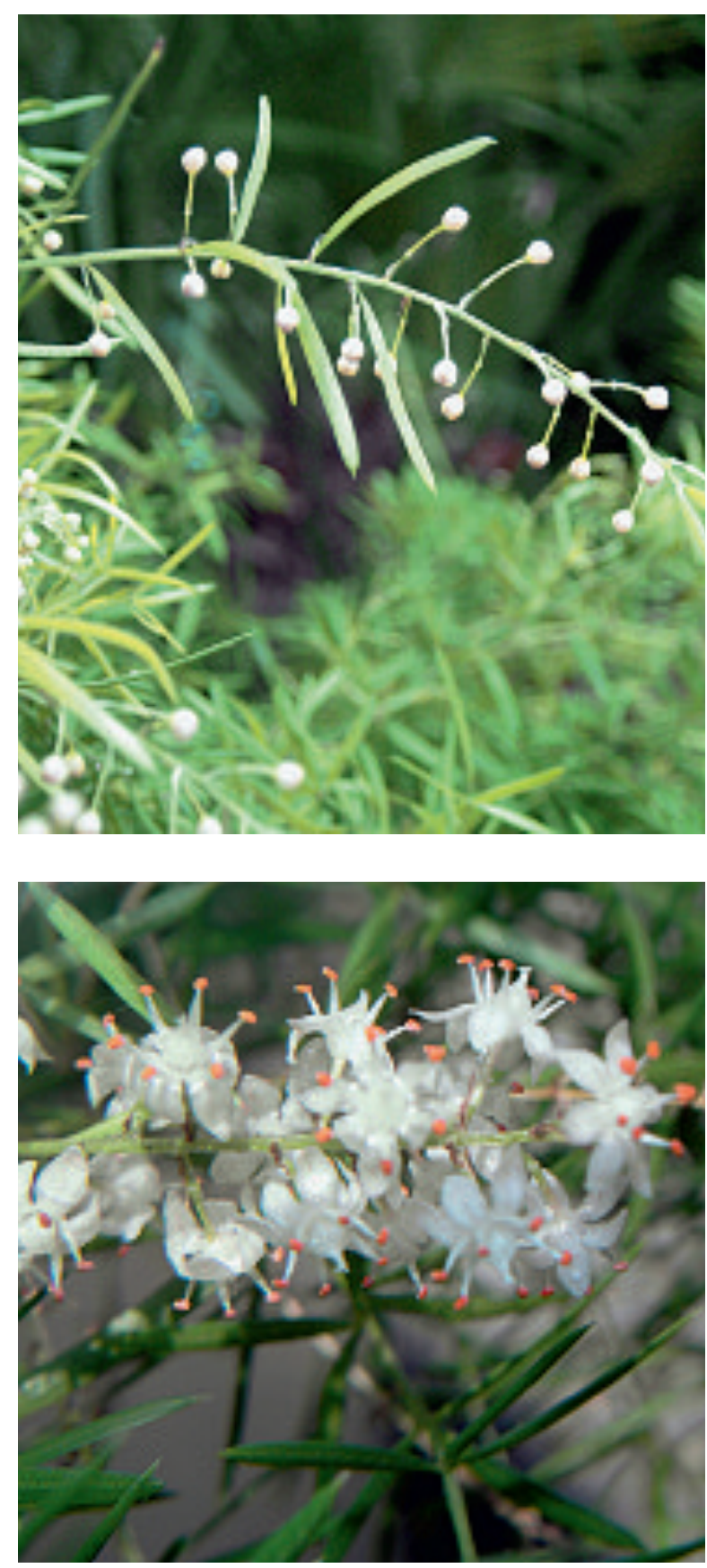


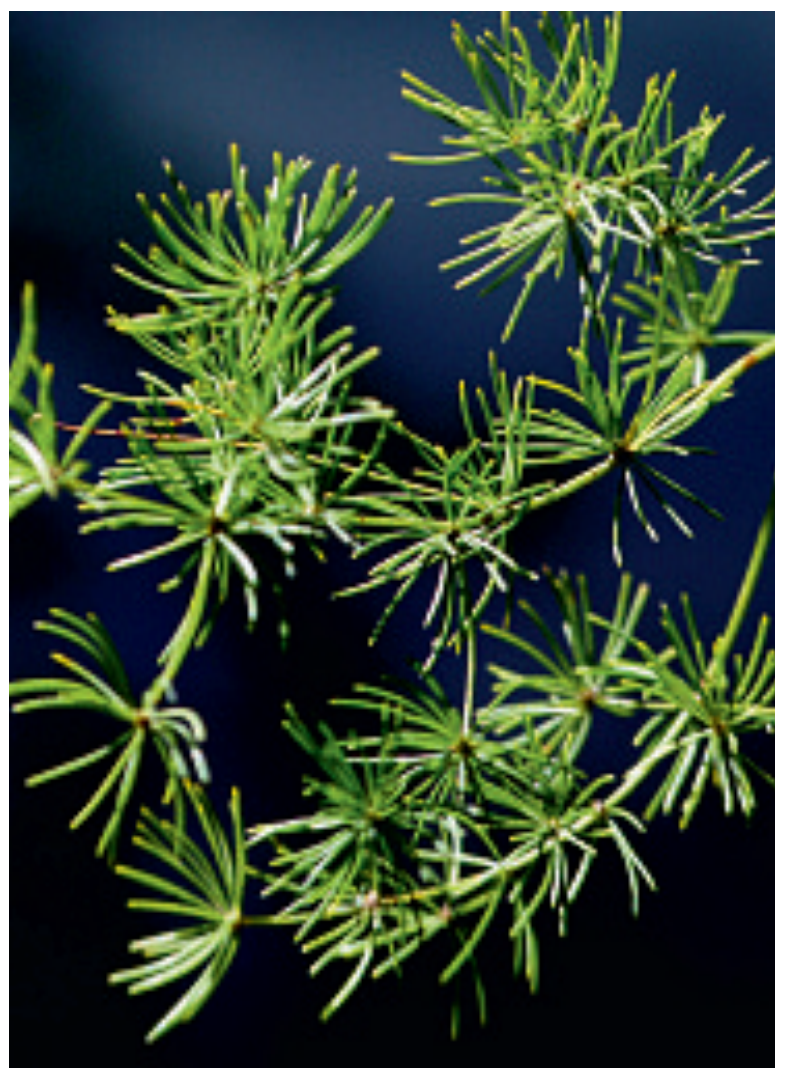

ten sind mehrere recht große Exemplare zu sehen, die im historischen Eingangsschauhaus sowie im Tropicarium im Bereich der trockenen Tropen gedeihen.

Asparagus densiflorus wurde um 1890 als Zierpflanze nach Europa eingeführt (Perry 1974). Es sind drei Varianten bzw. Sorten im Handel: die bekannte 'Sprengeri', zudem 'Meyer' (auch 'Meyersii') und 'Myriocladus. Dieser tropisch anmutenden Pflanze sieht man es gar nicht an, dass sie relativ kälteunempfindlich ist. In milderen Gebieten Deutschlands (z. B. im Rhein-Main-Gebiet) erträgt sie, im Freien ausgepflanzt, auch längere Kälteperioden recht gut. Auch Nachtfröste übersteht sie. Sehr strenge Winter wie die drei vorangegangenen überlebt sie allerdings draußen nicht.

Seine Wurzeln haben knollenförmig verdickte Abschnitte. Die grünen Triebe tragen schuppige, bleiche Blätter mit einer kleinen, nach unten gerichteten scharfen Spitze. Im ganz jungen Zustand erinnern die Triebe an dünne Sprosse des grünen Spargels. Zier-Spargel ist allerdings im Gegensatz zum Gemüse-Spargel giftig, zumindest für kleine Haustiere wie
Hunde und Katzen. Seine Zweige stehen entweder aufrecht, biegen sich elegant zur Seite oder krallen sich an Sträuchern und Bäumen fest.

\section{Spross}

Aus den Achseln der stacheligen Schuppenblätter entspringen Seitenzweige. Ihre Länge nimmt von der Basis des Hauptzweiges bis zu dessen Spitze hin ab. Sie tragen an Tannennadeln erinnernde Organe von frischgrüner Farbe, die der Laie als Blätter anzusehen geneigt ist. Es handelt sich dabei aber um blattartig abgeflachte Kurztriebe (Phyllokladien). Diese enthalten wie auch „richtige“ Blätter Chlorophyll und betreiben Fotosynthese. Sie sind größtenteils zu Büscheln von je 2-6 (meist 3) angeordnet, stehen aber auch einzeln. Die Zweigspitzen tragen häufig 4-9 in einem Wirtel angeordnete Phyllokladien. Ähnlich sind auch die Triebe des Gemüse-Spargels aufgebaut, sobald sie die Erde durchdrungen haben und ergrünt sind (siehe auch BeYHL 2006).

Leider bleibt auch Zier-Spargel nicht von Schädlingen verschont. Am Spross nisten sich gerne Schmierläuse und Spinnmilben ein, insbesondere, wenn die Pflanzen geschwächt sind. Auch Pilzbefall ist möglich.

\section{Blütenstände und Blüten}

Im Frühling und Sommer entwickelt ZierSpargel vielblütige, $2-5 \mathrm{~cm}$ lange, würstchenförmige, traubenartige Blütenstände. Sie bilden sich paarweise jeweils rechts und links am Seitenzweig in der Achsel schuppenförmiger Tragblätter.

Sie entwickeln sich aber auch einzeln in der Achsel von Tragblättern oder statt eines Phyllokladien-Büschels. Hin und wieder steht in einem solchen Blütenstand anstelle einer Blüte ein Phyllokladium. Umgekehrt sind bisweilen auch einzelne Blüten zwischen den Phyllokladi-

Abb. 3: Der Spross des in Südafrika heimischen ZierSpargels Asparagus retrofractus erinnert an einen Zweig eines Nadelbaumes. 
en oder anstelle eines einzeln stehenden Phyllokladiums anzutreffen. Phyllokladium, Phyllokladienbüschel, Einzelblüte und Blütenstand sind wechselseitig austauschbar.

Die Blütenstände umfassen zwischen 3 und 34, im Durchschnitt 12 gestielte Blüten. Da alle Blütenstiele annähernd gleich lang sind, entsteht die walzen- oder würstchenartige Gestalt des Blütenstandes, worauf sich der Art-Beiname (lat. densiflorus = dichtblütig) bezieht. Die ältesten Blüten stehen im unteren Teil der Infloreszens und blühen zuerst auf, es folgen die im Blütenstand weiter oben gelegenen Blüten. Besonders große Blütenstände entwickeln in ihrem untersten Teil noch zusätzlich eine oder mehrere blütentragende Seitentrauben, die ebenfalls von unten nach oben aufblühen. Einige Blütenstiele tragen außer der zugehörigen Blüte ein kurzes Seitenzweiglein. Die kleinen seitlichen Teilblütenstände erinnern an die Trauben der heimischen Schattenblume (Maianthemum bifolium).

Die zwittrigen Blüten sind weißlich gefärbt mit einem rosa Schimmer. Ihr pfirsichartiger Duft ist für bestäubende Insekten sehr verlockend. Die 6 Zipfel der glockenförmigen Perianthröhre sind zurückgeschlagen und die 6 weißen Staubgefäße tragen lebhaft orange gefärbte Pollensäcke. Der dicke Stempel, der in eine dreizipflige Narbe ausläuft, ist weiß gefärbt. Im Unterschied dazu sind die Blüten des Gemüse-Spargels grünlich.

Aufgrund von Unterschieden im Bau der Blütenstände und auch der Einzelblüten wird A. densiflorus gelegentlich von Asparagus abgetrennt und in die Gattung Protasparagus gestellt (vgl. Malcomber \& Demissew 1993).

\section{Früchte}

Die Früchte des Zier-Spargels sind kleine, giftige Beeren von 0,5-1,0 cm Durchmesser. Im unreifen Zustand sind sie grün, bei der Reife fär-

Abb. 4 (oben): Wildspargel wird vielerorts im Mittelmeergebiet für den Verzehr gesammelt.

Abb. 5 (unten): Asparagus densiflorus im Eingangsschauhaus des Palmengartens.
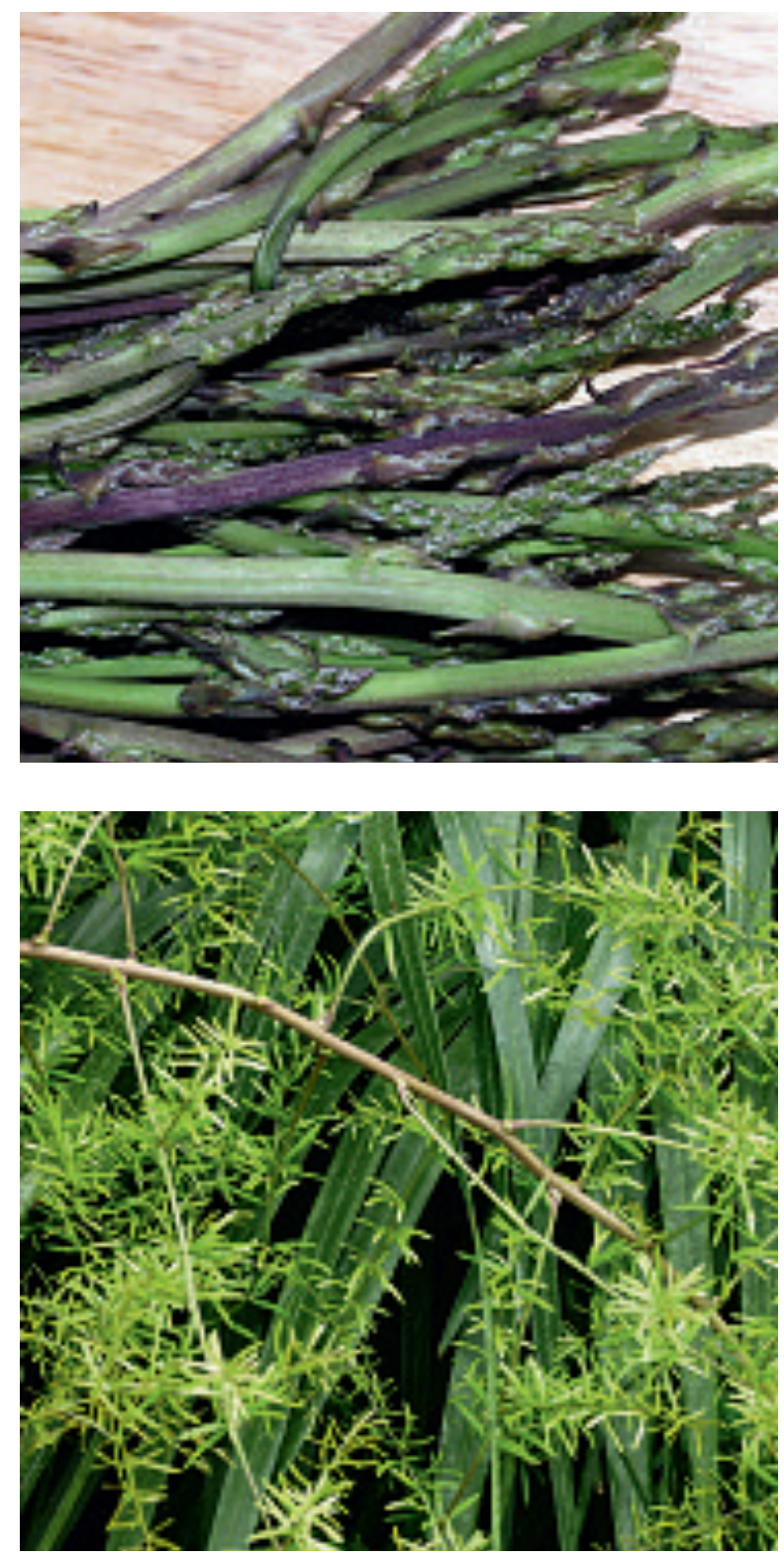

ben sie sich lebhaft rot. Sie enthalten einen, seltener mehrere Samen. Pro Blütenstand entwickeln sich etwa 1-3 Früchte, oft nur eine einzige. Viele der Blütenstände entwickeln überhaupt gar keine Früchte. Es werden also deutlich mehr Blüten gebildet, als befruchtet werden. Ähnliche Verhältnisse liegen beim Gemüse-Spargel vor. Die wenigen Beeren reichen aber offensichtlich aus, dass sich die beiden $\mathrm{Ar}$ ten erfolgreich ausbreiten können. So konnte sich z. B. Gemüse-Spargel von seinen Anbauflächen in der Oberrheinischen Tiefebene ausgehend als auffällige, weithin sichtbare Begleitpflanze entlang der Autobahn Frankfurt-Basel etablieren. Die bei uns als Zierpflanze geschätz- 


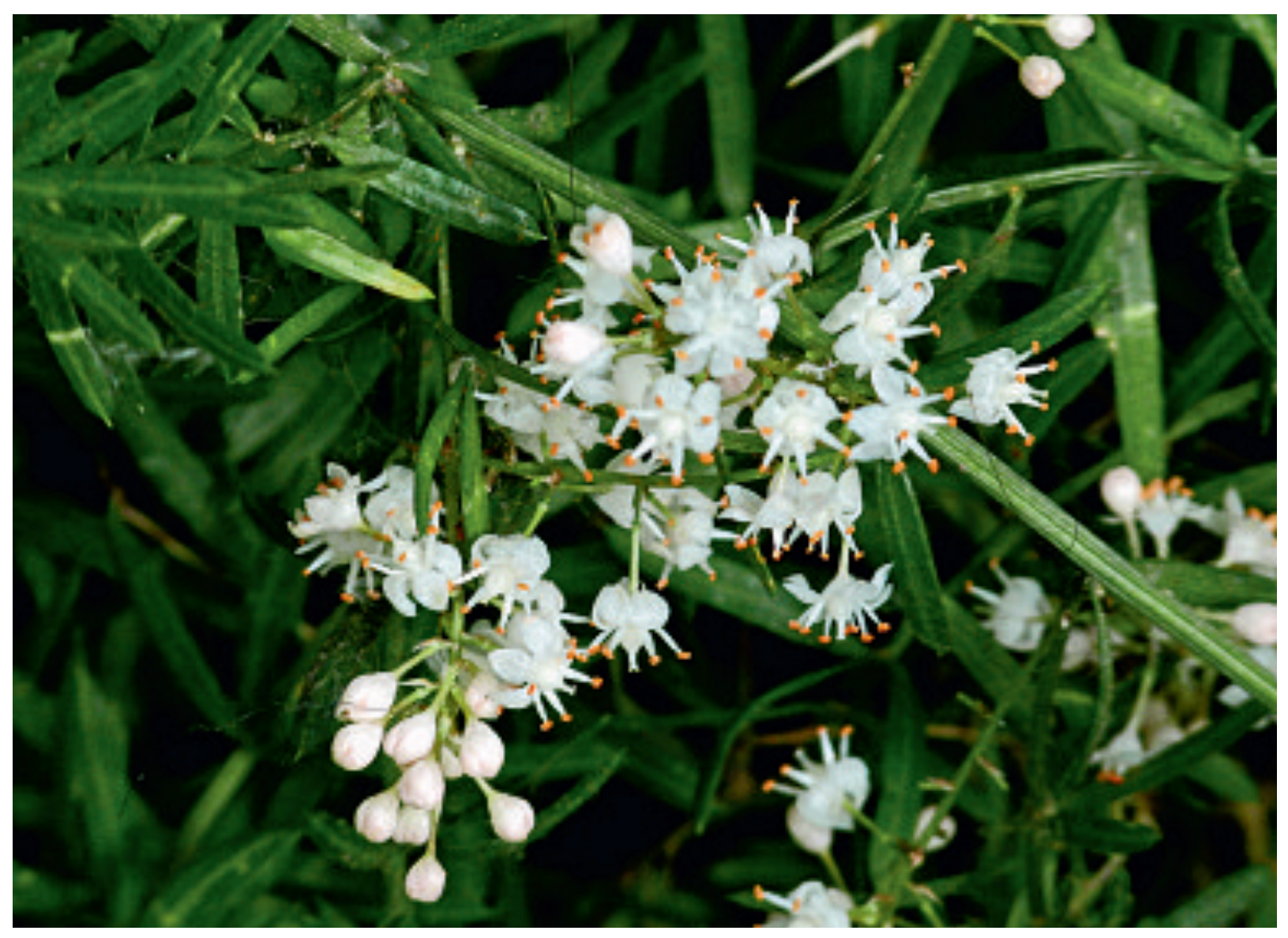

te Art A. densiflorus ist mittlerweile in Australien zusammen mit einigen anderen Vertretern der Gattung Asparagus zu einem äußerst lästigen und schwer bekämpfbaren Neophyten geworden (Bowden \& Rogers 1995, BatcheLOR \& SCOTT 2006).

\section{Literatur}

Batchelor, K. L., \& Scott, J. K. 2006: Review of the current taxonomic status and authorship for Asparagus weeds in Australia. - Plant Protection Quarterly 21: 128-130.

BeLl, A. D. 1994: Illustrierte Morphologie der Blütenpflanzen. - Stuttgart.

Beyнl, F. E. 2006: Morphometrische Untersuchungen am Gemüsespargel, Asparagus officinalis L. - Acta Biol.

Benrodis 13: 169-184.
Bowden, D., \& Rogers, R. W. 1995: Protasparagus densiflorus (aethiopicus): an environmental weed of coastal vegetation reserves. - Pacif. Conserv. Biol. 2: 293-298. Green, P. S. 1986: The correct name for Asparagus sprengeri. - Plantsman 7: 249-250.

Jessop, J. P. 1966: The genus Asparagus in Southern Africa. - Bothalia 9: 31-96.

Malcomber, S. T. \& Demissew, S. 1993: The status of Protasparagus and Myrsiphyllum in the Asparagaceae. Kew Bull. 48: 63-78.

Mevius, W. 1953: Taschenbuch der Botanik. Teil II. Systematik. - Stuttgart.

Perry, F. (Hrsg.) 1974: Complete guide to plants and flowers. - New York.

Schmeil, O. 1917: Leitfaden der Botanik. - Leipzig. Smalian, K. 1915: Grundzüge der Pflanzenkunde. Leipzig.
Abb. 6: Im historischen Eingangsschauhaus blüht Asparagus densiflorus regelmäßig. 